\title{
Sand und Wasser: Analogieexperimente zur Geologie im Schulunterricht
}

\author{
• hans.steyrer@sbg.ac.at, Fachbereich Geographie und Geologie, Universität Salzburg \\ ** sylke.hilberg@sbg.ac.at, Fachbereich Geographie und Geologie, Universität Salzburg \\ eingereicht am: 01.02.2016, akzeptiert am: 20.04.2016
}

\begin{abstract}
Die Vermittlung von Geologie ist eine große Herausforderung weil geologische Prozesse oft tief in der Erde oder nur sehr langsam ablaufen. Geologie ist zwar von großer gesellschaftlicher Bedeutung, spielt aber im Schulunterricht kaum eine Rolle. Modelle können Lernenden helfen, durch die direkte Beobachtung einige grundlegende geologische Prozesse zu erfassen. Grundwasser wird durch Modelle für die hydraulische Durchlässigkeit in porösen Medien thematisiert. Der Beitrag gibt eine detaillierte Beschreibung der Experimente und Lernziele.
\end{abstract}

Keywords: Lehre, Geologie, Hydrogeologie, Analogmodellierung

\section{Sand and water: analogue modelling of geological processes in school}

Teaching geology is a challenge because some geological processes are located below the earth's surface and occur with extremely low velocities. Although geological processes are of great social relevance, geology plays a minor role at school. Models that reflect long-term subsurface processes are required to familiarize pupils with geology. We describe easy to use models for basic geological processes and for groundwater flow in porous media and provide a detailed description of the experimental set-up and learning outcome.

Keywords: teaching, geology, hydrogeology, analogue modelling

\section{$1 \quad$ Einleitung}

Geologie ist beinahe allgegenwärtig in Form von Vulkanen, Erdbeben, Rohstoffen und fossilen Energieträgern. Vor allem in Österreich sind die Auswirkungen geologischer Prozesse in der Gestalt der Alpen sogar sehr offensichtlich und prägen den Lebensraum (Naturgefahren, Raumordnung) und den Wirtschaftsstandort Österreich (z. B. Tourismus) in erheblichem Maße.

Die Verfügbarkeit von sauberem Trinkwasser hängt neben klimatischen Bedingungen ganz wesentlich von den hydrogeologischen Eigenschaften der Gesteine ab, die das Niederschlagswasser speichern und in denen es vor Verunreinigungen geschützt ist.

Trotz dieser großen Bedeutung der Geologie im Alltag wird, wie ein Blick auf die Lehrpläne für Geographie und Wirtschaftskunde, wie auch für Biologie und Umweltkunde zeigt, die Geologie kaum im Schulunterricht thematisiert. Der Begriff Geologie taucht in den Lehrplänen für Geographie- und Wirt- schaftskunde (BM:UKK 2016a, b) nicht auf. Auch naheliegende Querverbindungen zwischen geographischen und geologischen Themen werden nicht angeführt. Beispielsweise wird der Themenbereich Rohstoffe nicht explizit hinsichtlich geologischer Aspekte beleuchtet oder die Fragen der Raumordnung nicht mit geologischen Naturgefahren verknüpft.

In den Lehrplänen für Biologie und Umweltkunde (BM:UKK 2016c, d) sollen „Grundlegende geologische Kenntnisse [...] dem Verständnis des Bodens und des Zusammenwirkens von belebter und unbelebter Natur dienen" (BM:UKK 2016c, 4). Sie werden in der dritten Klasse unter dem Oberbegriff Ökologie und Umwelt vermittelt. Einzig in der Oberstufe (6. Klasse) wird die Geologie unter dem Oberbegriff Bioplanet Erde vertiefend behandelt („Einblick in die Stellung der Erde im Weltall, Wissen um Aufbau und Struktur der Erde und der geodynamischen Formungskräfte als Grundlage der Entstehung ausgewählter österreichischer Landschaften") (BM:UKK 2016d, 3). 
Ein Grund für die geringe Präsenz geologischer Themen im Schulunterricht lässt sich darin finden, dass vor allem endogene geologische Prozesse nicht leicht zu beobachten sind. Selbst geologisch gesehen rasche Bewegungen, wie die Öffnung des Pazifischen oder des Atlantischen Ozeans, laufen „nur“ mit der Geschwindigkeit des Wachstums unserer Fingernägel $\mathrm{ab}$, manche Prozesse sind noch wesentlich langsamer. Viele geologische Vorgänge sind direkt überhaupt nicht beobachtbar, weil sie unter der Oberfläche, oft sogar tief in der Erdkruste oder im Erdmantel stattfinden. Wir sind nur mit den, im wahrsten Sinne des Wortes, oberflächlichen Phänomenen der Geologie konfrontiert, mit Vulkanausbrüchen oder geogenen Naturkatastrophen. Wir kennen die Ozeane, die 2/3 der Erdoberfläche bedecken und doch nur etwa $5 \%$ der 4,6 Milliarden Jahre Erdgeschichte für ihre Entstehung gebraucht haben, wie absolute Altersdatierungen der ältesten Ozeanböden belegen (z. B. Müller et al. 2008, mit zahlreichen Literaturhinweisen). Die Bildung der beeindruckenden Berge, Täler und Flüsse unserer Umgebung fand im Zeitalter des Quartärs (also in den letzten ca. 2,6 Millionen Jahren) statt, liegt somit nur im Promillebereich der Erdgeschichte.

Genau diese beiden Fakten - die Langsamkeit der geologischen Prozesse und ihre Verborgenheit in der Tiefe - war auch die Ursache dafür, dass die Geologie erst in der zweiten Hälfte des 19. Jahrhunderts zu einer modernen naturwissenschaftlichen Denkweise gefunden hat. $\mathrm{Zu}$ verlockend war es offenbar, geologische Phänomene im Rahmen der Schöpfungsgeschichte zu erklären: Die Sintfluthypothese für erratische Blöcke oder die Erklärungen, wie Muscheln auf einen Berg kommen (Cutler 2004) sind nur zwei von zahlreichen Beispielen. Oft wurde auch auf naheliegende, uns aus anderen Lebensbereichen vertraute Konzepte zurückgegriffen, wie etwa die Vorstellungen von der Erde als Organismus, der Meerwasser „verdaut“ und als Süßwasser an Quellen ausscheidet - eine Vorstellung, die in der Antike und bis ins Mittelalter hinein vertreten wurde (Hölting \& Coldewey 2013). Auf derartige Lösungsansätze sind wohl teilweise noch heute weit verbreitete Konzepte zu bestimmten, nicht direkt beobachtbaren geologischen Prozessen zurückzuführen. Dazu gehört z. B. die Vorstellung der Wasserader, die bei der Beschreibung von Grundwasser gern bemüht wird (Reinfried et al. 2012; Unterbruner et al. 2015).

Laborversuche, die geologische Prozesse mehr oder weniger maßstabsgetreu nachbilden, sind schon seit Beginn des 20. Jh. erfolgreich angewendet worden (Riedel 1929; Hubbert 1937), um den Faktor Zeit auf ein direkt beobachtbares Maß zu skalieren und haben zu spektakulären Ergebnissen geführt (z. B. Tapponnier et al. 1986). Sie haben bis heute ihre Bedeutung für die Erklärung komplexer geologischer Abläufe behalten.
Eigenständige Beobachtungen in der Natur, etwa im Zuge einer Wanderung entlang der Ufer eines Flusses, wo der Unterschied zwischen den groben Sedimenten im Hauptgerinne und den feinkörnigen Sanden der Kehrwasserbereiche offensichtlich ist, können mit einem einfachen Versuch auf physikalische Grundprinzipien zurückgeführt werden - dieser Zusammenhang wurde erst 1935 mathematisch-physikalisch formuliert, übrigens im Zuge einer Dissertation (Hjulström 1935).

Diese junge aktualistische Denkweise, die für die Geologie im 19. Jh. noch neu war und heute selbstverständlicher Schlüssel für geologische Prozesse ist, kann auf viele Beobachtungen angewendet werden und soll Anregung sein, eigene Modellversuche zu entwickeln.

\section{Modelle in der universitären Lehre und im Schulunterricht}

Auch für Fachstudierende der Geologie, die generell die Befähigung mitbringen sollten, sich räumlich und zeitlich nicht direkt beobachtbare Prozesse vorzustellen, sind Analogmodelle im oben beschriebenen Sinne hilfreich, um abstrakten Vorgängen eine greifbare, weil bildliche Form zu verleihen. In der universitären Ausbildung werden daher verschiedenste Modelle verwendet.

Diese Modelleinrichtungen, z. B. die Lehner'sche Sandbox (Steyrer 2009) oder das Grundwassermodell (Ecovia 2016), erfordern einen technischen Aufwand, der an Schulen schwer umsetzbar ist, daher stellen wir im Folgenden einige einfache Versuche vor, die mit einfachen technischen Mitteln, leicht verfügbaren Materialien und geringem Zeitaufwand die Möglichkeit bieten, geologische Prozesse zu beobachten, eigene Schlüsse daraus zu ziehen und Parallelen in der Natur aufzuzeigen.

Generell bietet sich bei der Arbeit mit Modellen in der universitären Lehre wie auch im Schulunterricht eine gute Möglichkeit, Inhalte nicht in Form von Frontalunterricht vorzuführen, sondern gemeinsam mit Schülerinnen und Schülern zu erarbeiten. Anhand der Modelle werden gemeinsam mit den Lernenden Hypothesen über den Verlauf und das Ergebnis von Modellversuchen aufgestellt. Es können hierbei auch vorhandene Präkonzepte aufgegriffen werden. Im Sinne von "conceptual change“ (z. B. Strike \& Posner 1992; Sinatra 2005) können einfache Modelle kognitive Konflikte bezüglich vorhandener Präkonzepte hervorrufen und damit Lernende dazu bewegen, ihre dadurch offensichtlich inadäquaten Vorstellungen zu überdenken und das korrekte Konzept zu übernehmen. 
Die im Folgenden vorgestellten vereinfachten Modelle für den Schulunterricht eignen sich dafür, dass Schülerinnen und Schüler sie selbständig in Kleingruppen, z. B. auch in Form eines Stationenbetriebs zum Thema Geologie mit Hilfe von Arbeitsanweisungen durchführen und ihre Beobachtungen anschließend in der Gruppe oder im Plenum diskutieren.

\section{Versuche}

\subsection{Versuch 1: Entstehung von Schichtung in Sedimenten}

Schichtung ist für die meisten Sediment- oder Ablagerungsgesteine charakteristisch und kommt durch wechselnde Ablagerungsbedingungen zustande. In klastischen Sedimenten bestimmt die Fließgeschwindigkeit des Transportmediums Wasser, welche Korngröße noch transportiert werden kann, bzw. welche Korngrößen liegen bleiben, also abgelagert werden. Das Hjulström-Diagramm (Hjulström 1935) veranschaulicht die Beziehung zwischen mittlerer Korngröße, Fließgeschwindigkeit und Kornverhalten: je größer die Fließgeschwindigkeit, desto größer kann das transportierte Korn sein. Die Entstehung einer Schichtung in klastischen Sedimenten durch abnehmende Strömungsgeschwindigkeit kann mit dem folgenden Versuch visualisiert werden (Anm.: dieser Versuch wurde ursprünglich von einem der Autoren für die einführende Geologie Vorlesung an der Universität Salzburg konzipiert).

Geräte und Material: Becherglas mit mindestens 1 Liter Inhalt, Kochlöffel, beliebiges Gemisch aus verschieden groben Sanden, Schluff und Ton. Sowohl das Becherglas als auch das Sedimentgemisch kann mehrfach verwendet werden.

Durchführung (Abb. 1): Das Sand-Schluff-Tongemisch wird im Becherglas mit Wasser versetzt. (a) Mit dem Kochlöffel wird durch Rühren eine (kreisförmige) Strömung erzeugt. Nach dem Ende des Rührens kommt es zur Sedimentation, die durch das Becherglas sichtbar ist. (b) Bei nachlassender Strömungsgeschwindigkeit werden zuerst die größeren Körner sedimentiert, danach nimmt die Korngröße kontinuierlich ab, es entsteht eine sogenannte gradierte Schichtung. Die Schluff- und Tonfraktion bleibt Stunden bis Tage in Schwebe (Suspension) und bildet den Abschluss der Schichtung nach oben.

Ergebnis: Eine korngrößenabhängige Schichtung des Sediments wird erzeugt, der Zusammenhang zwischen Strömungsgeschwindigkeit und transportierbarer Korngröße wird sichtbar.
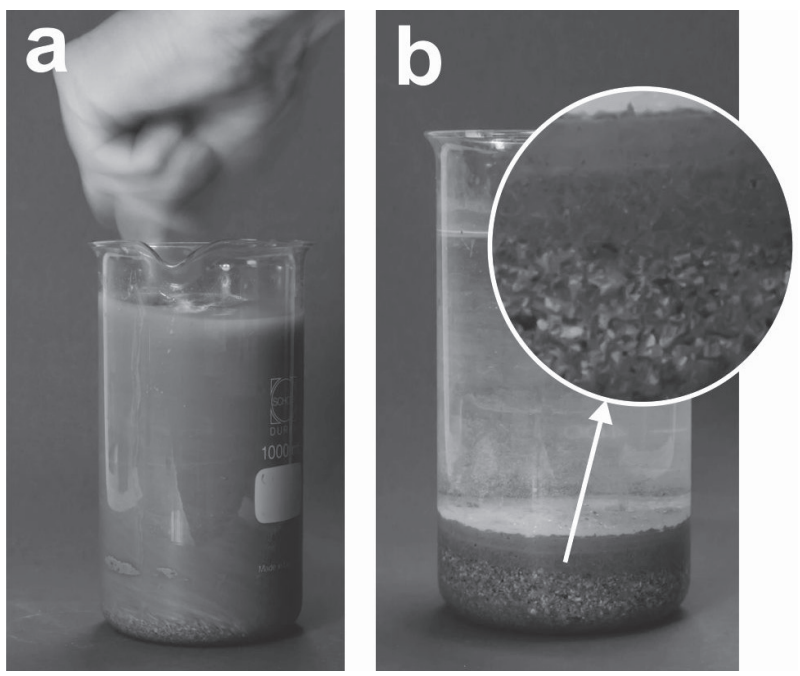

Abb. 1: Durchführung des Versuchs 1

\subsection{Versuch 2: Böschungswinkel in Lockergestei- nen}

Natürliche Böschungen, oft auch als Hänge bezeichnet, entstehen durch das Zusammenwirken verschiedener geologischer Prozesse, wie Hebung durch Gebirgsbildung, Erosion und Sedimentation. Sie werden oft durch Straßeneinschnitte oder andere Bauwerke künstlich überprägt, wobei die Stabilität einer solchen Böschung von großer Bedeutung für den Bestand der Bauwerke und für die Sicherheit der Menschen ist. Die mögliche Steilheit und damit die Stabilität solcher Böschungen hängen von verschiedenen Faktoren ab. Ein häufiger Einflussfaktor ist der Wassergehalt: Bei ansonsten gleichen Bedingungen ist der natürliche Böschungswinkel im trockenen Zustand deutlich höher als im wassergesättigten Zustand. Der Unterschied wird im folgenden Versuch deutlich (Anm.: dieser Versuch wurde ursprünglich von einem der Autoren für die einführende Geologie Vorlesung an der Universität Salzburg konzipiert).

Geräte und Material: Quaderförmiges Glasgefäß mit ca. 10 Litern Inhalt (z. B. ein kleines Aquarium), $1 / 2$ kg Putzsand, Kartonschütte, Wasser. Alle Geräte und Materialien können beliebig oft wiederverwendet werden.

Durchführung (Abb. 2): (a) Mittels der Kartonschütte wird Sand in das trockene Aquarium geschüttet. Es bildet sich ein konstanter Böschungswinkel von $32^{\circ}$ aus (b), wie er für natürliche Böschungen in trockenen Lockergesteinen charakteristisch ist und wie er an vielen Stellen in der Natur beobachtet werden kann. (c) Der Versuch wird unter Wasser wiederholt, es bildet sich ein wesentlich niedrigerer Böschungswinkel von $13^{\circ}$ aus.

Ergebnis: Der Böschungswinkel von Lockergesteinen hängt wesentlich vom Wassergehalt ab. Für Bau- 

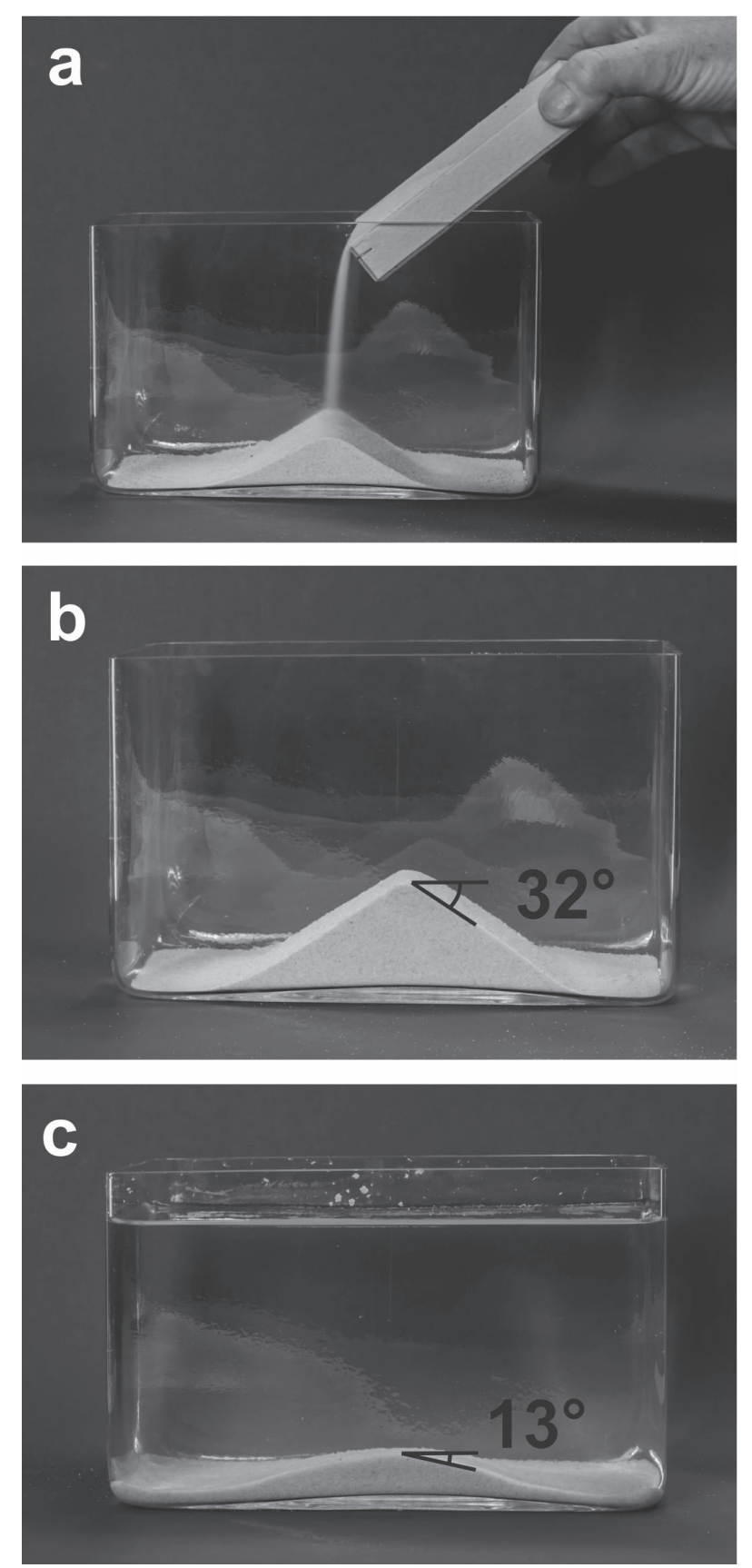

Abb. 2: Durchführung des Versuchs 2

maßnahmen ist der folgende Zusammenhang wichtig: Der mögliche Böschungswinkel und damit die Stabilität einer Böschung kann erhöht werden, wenn der Wassergehalt reduziert wird (z. B. durch Drainage).

\subsection{Versuch 3: Bildung einer Störung im Modell- maßstab}

Störungen entstehen in der spröden Erdkruste immer dann, wenn sich Lithosphärenplatten gegeneinander bewegen. Solche Störungen haben oft nur Dimensionen im Meterbereich, können aber auch Tausende Kilometer lang sein. Große Störungen, wie die nordanatolische oder die San Andreas Störung oder, sucht man einen regionalen Bezug auch die periadriatische Naht, verursachen immer wieder Erdbeben und beeinflussen damit direkt das Leben der Menschen im Wirkungsbereich dieser geologischen Phänomene. Im Modellversuch kann gezeigt werden, wie die Erdkruste auf Bewegungen reagiert und welche Oberflächenstrukturen entstehen. Sand ist als Analogmaterial für die spröde Erdkruste seit langem gebräuchlich, da er viele Eigenschaften der Erdkruste - verkleinert auf den Modellmaßstab - zeigt (z. B. Riedel 1929; Hubbert 1937). Der hier vorgestellte Versuch ist eine vereinfachte Version der bereits vorgestellten Analogmodelle (Lehner'sche Sandbox) zum Verständnis plattentektonischer Prozesse (Steyrer 2009).

Geräte und Material: zwei Kartonplatten DIN A2, 2-3 kg Putzsand, zwei $1 \mathrm{~m}$ lange Holzleisten $(3 \mathrm{~cm} \mathrm{x}$ $3 \mathrm{~cm}$ ), 1 Flächenspachtel mit $30 \mathrm{~cm}$ Breite, eine Büroleuchte. Alle Geräte und Materialien können beliebig oft wiederverwendet werden.

Durchführung (Abb. 3): (a) Die Kartons werden überlappend angeordnet. (b) Sand wird aufgebracht, (c) mit Hilfe der Holzleisten und der Spachtel wird ein Sandkörper mit ebener Oberfläche erzeugt. (d) Die Kartons werden langsam gegeneinander verschoben (entspricht in diesem Fall einer dextralen Seiten- oder Blattverschiebung), es bildet sich neben der Hauptstörung eine Reihe charakteristischer Nebenstörungen, die sogenannten Riedel- und Antiriedelstörungen ( $\mathrm{R}$ und R' in Abb. 3e; im Streiflicht einer Schreibtischlampe werden die Strukturen klar erkennbar), benannt nach W. Riedel, der diesen Zusammenhang in seiner Dissertation erarbeitet hat (Riedel 1929).

Ergebnis: An der Grenze der beiden Kartons bildet sich eine Störungszone aus, die beispielsweise mit den Oberflächenstrukturen der San-Andreas Störung (Kalifornien) sehr schön verglichen werden kann (Abb. 3e, rechts).

\subsection{Versuche 4 und 5: Eigenschaften und Prozes- se in Porengrundwasserkörpern}

Das Fließen von Grundwasser in Lockersedimenten ist auf die Porenräume beschränkt, die sich zwischen einzelnen Körnern bilden. Derartige Grundwasserleiter werden daher auch als Porenaquifere bezeichnet und von solchen Grundwasserkörpern unterschieden, die sich in Festgesteinsklüften oder in Karsthöhlen bilden (Hölting \& Coldewey 2013).

Um Grundwasser bilden zu können, müssen die Porenräume einerseits miteinander in Verbindung stehen, andererseits müssen die Poren ausreichend groß sein, um nicht vollständig vom die Kornoberflächen benetzendem Haftwasser erfüllt zu sein. Der Porenanteil, auf den diese beiden Eigenschaften zutreffen, wird auch als effektives Porenvolumen bezeichnet, das 

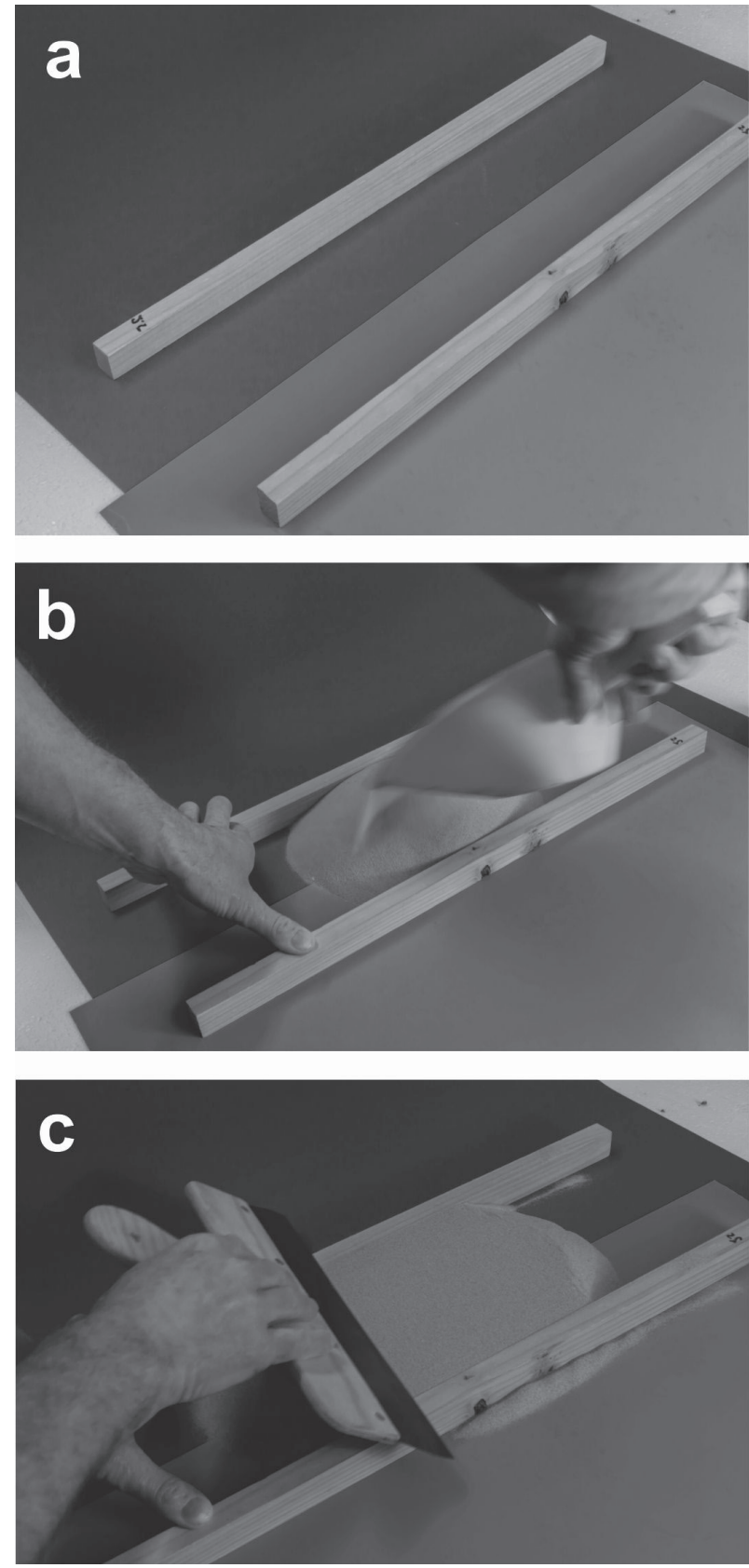

je nach Korngrößenverteilung im Sediment deutlich vom Gesamtporenvolumen abweichen kann (Davis $\&$ Wiest 1966). In der Natur lässt sich dieser Unterschied sehr gut bei Starkniederschlägen auf ebenen Grünflächen nachvollziehen. Ist der Untergrund darunter eher grobkörnig aufgebaut, versickert Regenwasser direkt, es bleibt kein Wasser an der Oberfläche zurück. Der größte Teil des Niederschlags wird zu Grundwasser. Ist der Untergrund jedoch aus sehr feinkörnigem Material (Ton oder Lehm) aufgebaut, bleibt Regenwasser lange an der Oberfläche stehen.
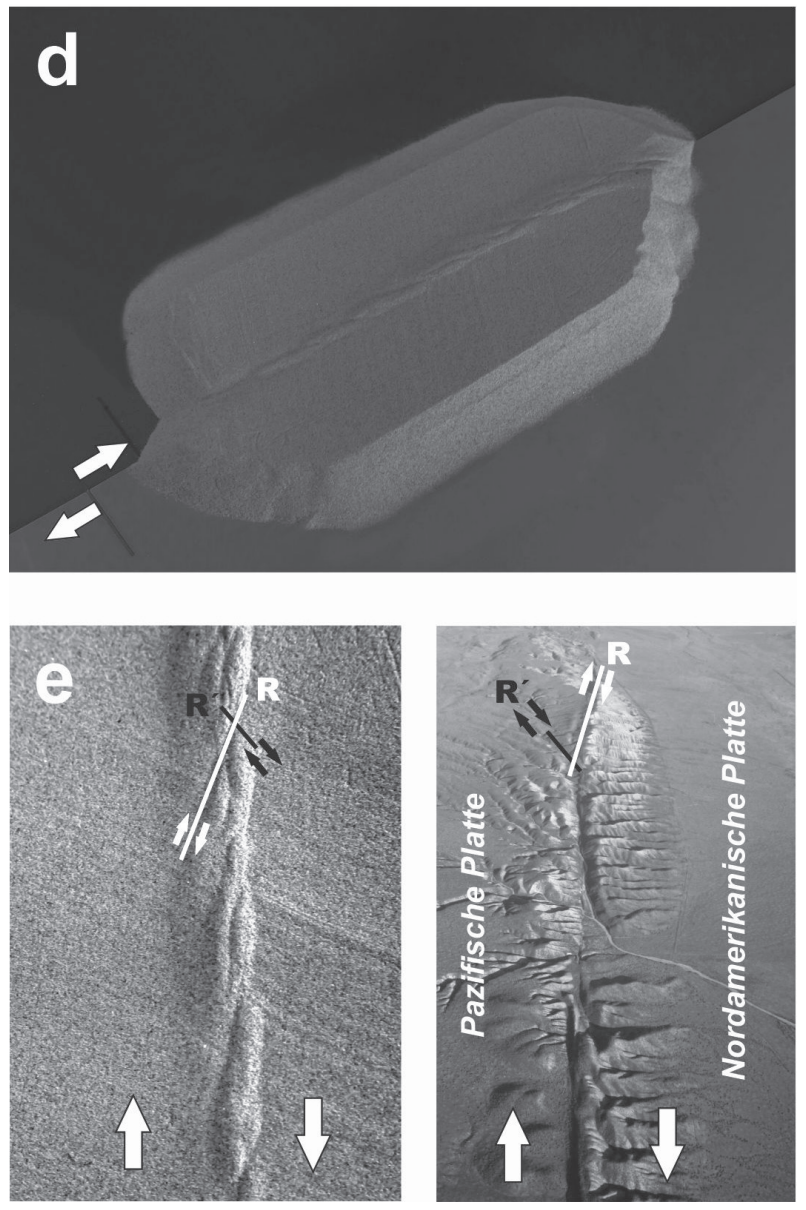

Abb. 3: Durchführung des Versuchs 3

\subsubsection{Versuch 4: Korngrößenverteilung und Poro- sität}

Der Versuch 4 (inkl. Variante) basiert auf dem Grundwassermodell nach Ecovia (2016), in dem Materialien verschiedener Korngrößen in einem quaderförmigen Plexiglasgefäß in Schichten eingebracht und in verschiedenen Versuchsanordnungen von Wasser durchströmt werden können, um den Zusammenhang von Korngröße und wassererfülltem Porenvolumen zu zeigen. Der hier vorgestellte Versuch wurde von einer der Autoren gegenüber der Vorlage einerseits hinsichtlich der verwendeten Materialien stark vereinfacht, andererseits aber zur Verdeutlichung von Wassermengen erweitert, in dem, anders als im Modell Ecovia, mit 

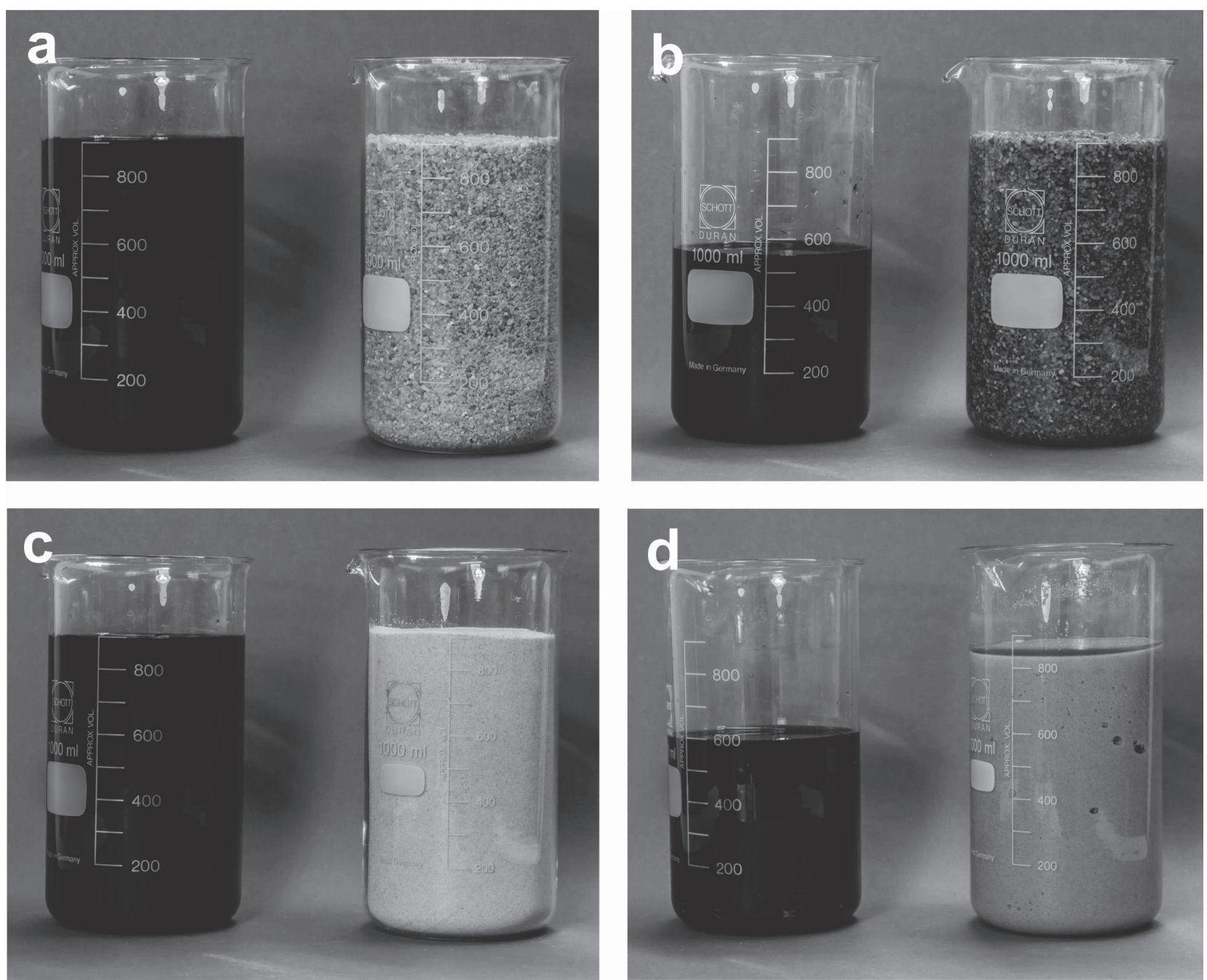

Abb. 4: Durchführung von Versuch 4

skalierten Gefäßen gearbeitet wird und die versickernden Wassermengen in den verschiedenen Medien direkt verglichen werden.

Material: Drei gleich große, skalierte Gefäße (z. B. $1000 \mathrm{ml}$ Bechergläser), gleichkörniger Grobsand bis Kies, gleichkörniger Fein- bis Mittelsand, Wasser, zur besseren Visualisierung der Prozesse mit Lebensmittelfarbe eingefärbt. Alle Materialien, mit Ausnahme der Lebensmittelfarbe, können beliebig oft wieder verwendet werden.

Durchführung (Abb. 4): Bechergläser werden mit Kies (a) bzw. Feinsand (c) gefüllt und anschließend mit gefärbtem Wasser übergossen, bis der gesamte Porenraum mit Wasser aufgefüllt ist, also eine geringe Menge an Wasser an der Oberfläche stehen bleibt (b und d). Über das verbleibende Restwasser kann die jeweils dazu benötigte Wassermenge festgestellt werden.

Durchführung Variante (Abb. 5): In einer erweiterten Variante werden Sand und Kies zu gleichen Teilen gemischt (a), wobei aus zweimal $450 \mathrm{ml}$ gleichkörnigem deutlich weniger als $900 \mathrm{ml}$ Mischmaterial werden (b). Das Gemisch wird komplett mit Wasser aufgefüllt und die dafür benötigte Wassermenge bestimmt (c).

Ergebnis: Grobsand kann rasch mit etwa $350 \mathrm{ml}$ Wasser angefüllt werden, bevor das Wasser oberhalb der Kiesschicht stehen bleibt - das effektive Porenvolumen beträgt damit hier $39 \%$. Das Gefäß mit Feinsand kann mit etwa $320 \mathrm{ml}$ Wasser gefüllt werden - das effektive Porenvolumen beträgt $36 \%$. Das effektive Porenvolumen ist zwar nur wenig geringer als in der Grobsandfraktion, allerdings fließt das Wasser hier langsamer durch die deutlich engeren Hohlräume - es ergeben sich vergleichsweise weitere Fließwege. Die hydraulische Durchlässigkeit (siehe auch Versuch 5) ist geringer, d. h. im gleichen Zeitraum fließt weniger Wasser durch dieses feine als durch das gröbere Material.

Im Gefäß, in das zweimal $450 \mathrm{ml}$ Material eingebracht wurden, befinden sich nun deutlich weniger als $900 \mathrm{ml}$ der Mischung aus Grob- und Feinsandfraktion, weil die kleineren Sandkörner in den Porenräumen zwischen den Kieskörnern Platz finden. Das Gefäß kann mit etwa $250 \mathrm{ml}$ Wasser gefüllt werden - das 

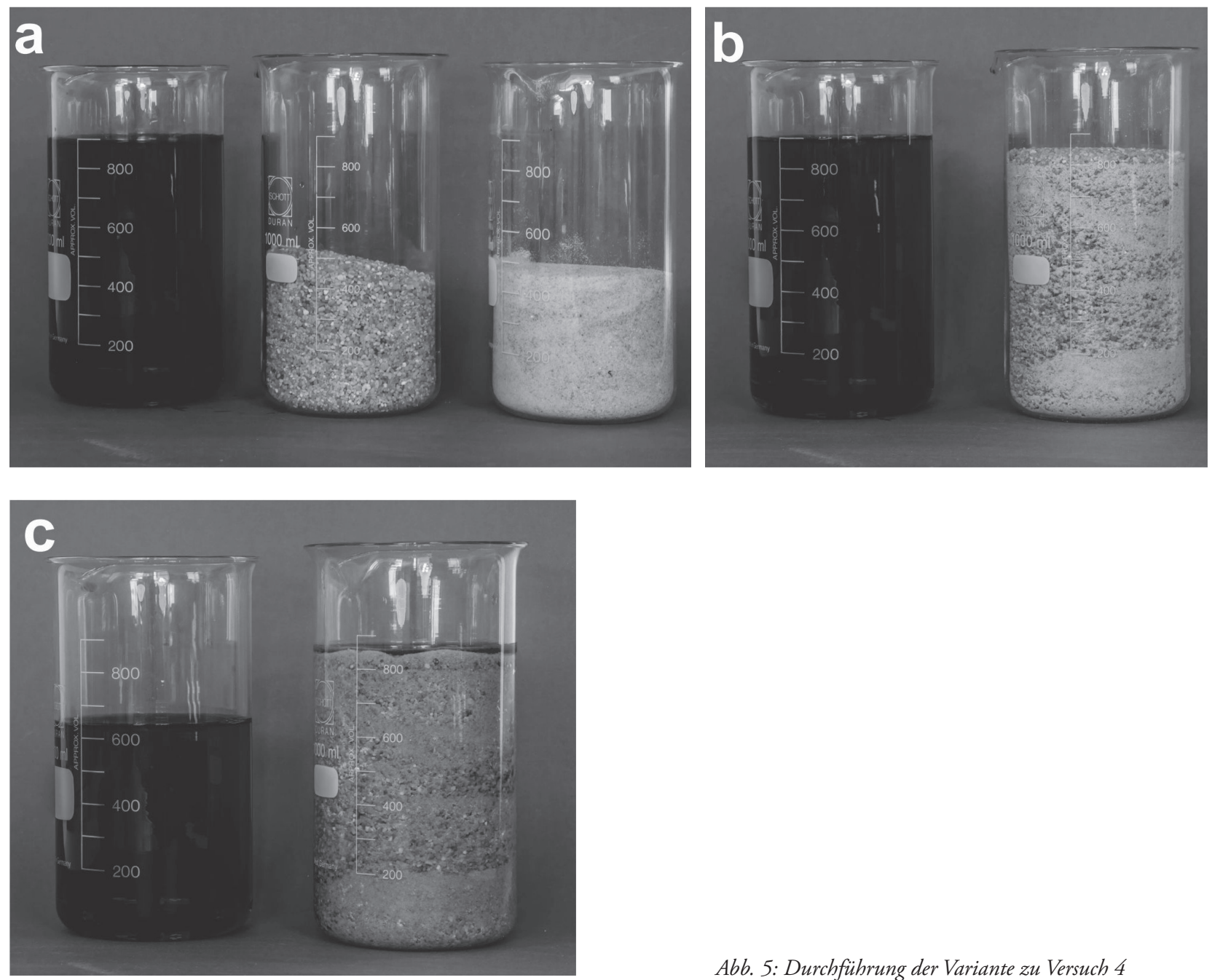

Abb. 5: Durchführung der Variante zu Versuch 4

effektive Porenvolumen beträgt $28 \%$ und liegt damit deutlich unter jenem der gleichkörnigen Materialien, weil ein großer Teil der Poren im gleichkörnigen Sediment hier bereits mit kleineren Körnern verfüllt ist.

\subsubsection{Versuch 5: Wasserdurchlässigkeit verschiede- ner Sedimente}

Der Versuch stellt eine gängige Visualisierung von Versickerungsprozessen in der universitären Lehre zur Hydrogeologie dar und wurde z. B. von Unterbruner \& Hilberg (2012) als Lehrmaterial für die Zielgruppe der 10 bis 15 jährigen vorgestellt.

Material: Drei gleich große Glasgefäße, gleichkörniger Grobsand bis Kies (z. B. Rasenquarz), gleichkörniger Fein- bis Mittelsand (Putzsand), Ton, Wasser, zur besseren Visualisierung der Prozesse mit Lebensmittelfarbe eingefärbt, drei skalierte Glasgefäße

Durchführung (Abb. 6): Die Gefäße werden mit jeweils der gleichen Menge an Ton, Feinsand und Grobsand oder Kies gefüllt. Es werden drei weitere skalierte Glasgefäße mit der jeweils gleichen Menge an gefärbtem Wasser gefüllt (a). Beim gleichzeitigen
Befüllen der drei Gefäße (b) mit gefärbtem Wasser zeigt sich, dass im Kies (links) das Wasser sofort versickert und die Porenräumen von unten her auffüllt, im Sand (Mitte) versickert das Wasser nur langsam. Im Ton (rechts) bleibt das Wasser bis zum Versuchsende an der Oberfläche und versickert nicht (c).

Ergebnis: Neben der effektiven Porosität spielt auch die Größe der einzelnen Hohlräume eine wichtige Rolle dabei, wie schnell das Wasser im Untergrund fließen kann. Je größer die Hohlräume sind, umso weniger Widerstand bieten die Körner für das Grundwasserfließen. Die Wasserteilchen müssen weniger Umwege machen, die Fließwege sind kürzer. Im gleichen Zeitraum kann durch den gleichen Fließquerschnitt im grobkörnigen Medium mehr Wasser fließen als im feinkörnigen Sand. Der Kies hat damit eine höhere hydraulische Durchlässigkeit. Im Ton sind die Hohlräume so klein, dass die Wasserteilchen nicht mehr hindurch passen oder wenn, dann nur sehr langsam darin fließen können. Dieses Material besitzt eine sehr geringe hydraulische Durchlässigkeit und kann als annähernd wasserdicht bezeichnet werden. Der Zusammenhang zwischen Korngröße und 

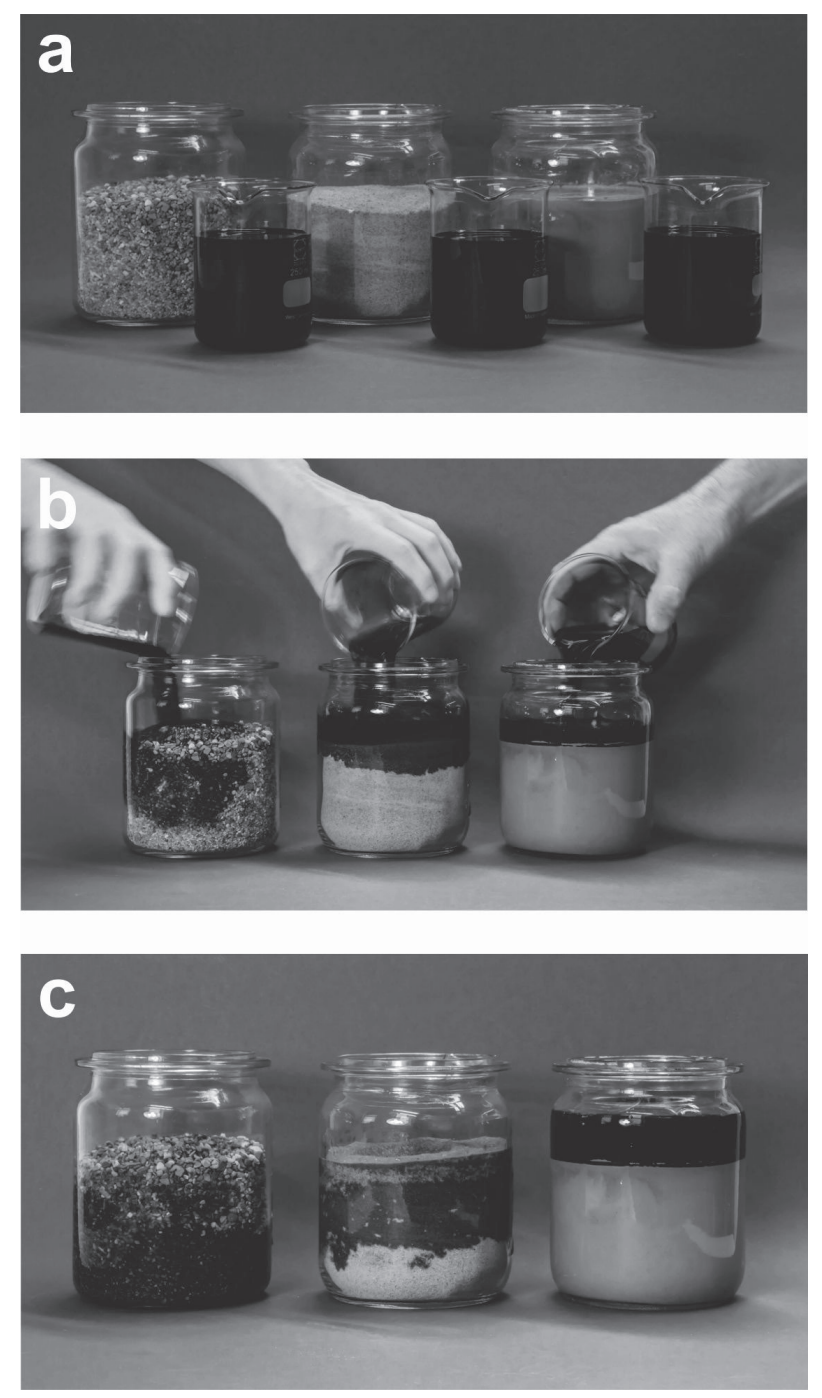

Abb. 6: Durchführung von Versuch 5

Durchlässigkeit wurde schon vor mehr als 150 Jahren erkannt (Darcy 1856).

\section{Schlussfolgerungen}

Die beschriebenen Modellversuche aus der Geologie und Hydrogeologie geben Anregungen, wie mit einfachen Mitteln Prozesse dargestellt und erklärt werden können, die in der Natur nicht direkt beobachtbar sind, weil sie - dem Wesen der Geologie entsprechend - unter der Erdoberfläche oder sehr langsam ablaufen und daher für den Menschen nicht direkt erfassbar sind. Wir kehren damit zurück zu den Anfängen der Geologie im 18. Jh. und dem Prinzip des Aktualismus: Geologische Prozesse, die heute ablaufen, sind zu allen Zeiten so abgelaufen, weil auch sie den gültigen physikalischen und chemischen Prinzipien unterworfen sind. Diese Denkweise war revolutionär, einfach nachzuvollziehen und äußerst erfolgreich - hat sie doch die Geologie erst zu einer modernen Natur- wissenschaft werden lassen und unser Verständnis für geologische Prozesse entwickelt.

Diese Prozesse sind im Alltag relevant, betreffen sie doch gesellschaftlich-zivilisatorische Aspekte wie Naturgefahren, Stabilität von Hängen und Bauwerken, Verfügbarkeit von Trinkwasser und vieles mehr. Abseits von Frontalunterricht kehren wir mit unseren Versuchen in einem anregenden und interaktiven Prozess zwischen Lernenden und Lehrpersonen zu den Wurzeln der Geologie zurück - wir arbeiten mit Sand und Wasser.

\section{Literatur}

BM:UKK -Bundesministerium für (2016a): AHSUnterstufe: Lehrplan Geographie und Wirtschaftskunde. https://www.bmbf.gv.at/schulen/unterricht/lp/ ahs9_784.pdf?4dzgm2 (31.03.2016)

BM:UKK (2016b): AHS Oberstufe: Lehrplan Geographie und Wirtschaftskunde. https://www.bmbf.gv.at/schulen/ unterricht/lp/lp_neu_ahs_06_11858.pdf?4dzgm2 (31.03.2016)

BM:UKK (2016c): AHS-Unterstufe: Lehrplan Biologie und Umweltkunde. https://www.bmbf.gv.at/schulen/ unterricht/lp/ahs5_779.pdf?4dzgm2 (31.03.2016)

BM:UKK (2016d): AHS-Oberstufe: Lehrplan Biologie und Umweltkunde. https://www.bmbf.gv.at/schulen/ unterricht/lp/lp_neu_ahs_08_11860.pdf?4dzgm2 (31.03.2016)

Cutler, A. (2004): Die Muschel auf dem Berg: Über Nicolaus Steno und die Anfänge der Geologie. München: Verlag A. Knaus.

Darcy, H. (1856): Les fontaines publiques de la ville Dijon. Paris.

Davis N.S. \& R.J.M. Wiest de (1966): Hydrogeology. Elsevier, Amsterdam.

Ecovia (2016) Demokoffer Grundwasser. http:// www.ecovia.ch/images/dokumente/demokoffer grundwasser/\%20gw-koffer-flyer_2015_d_klein.pdf (28.01.2016)

Hölting B. \& W.G. Coldewey (2013): Hydrogeologie. 8. Auflage, Stuttgart, Heidelberg: Springer Spektrum,.

Hjulström F. (1935): Studies of the morphological activity of rivers as illustrated by the River Fyris. Inaugural dissertation, Univ. Uppsala.

Hubbert, M.K. (1937): Theory of scale models as applied to the study of geologic structures. Geological Society Of America. Bulletin 48, 1459-1520.

Müller, R.D., M. Sdrolias, C. Gaina \& W.R. Roest (2008): Age, spreading rates, and spreading asymmetry of the world's ocean crust. Geochemistry Geophysics Geosystems 9, Q04006.

Reinfried S., S. Tempelmann \& U. Aeschbacher (2012): Adressing secondary school students' everyday idea about freshwater springs in order to develop an instructional tool to promote conceptual reconstruction. Hydrology Earth System Sciences 16, 1365-1377 
Riedel, W. (1929): Zur Mechanik geologischer Brucherscheinungen. Centralblatt für Mineralogie, Geologie und Paläontologie 1929, 354-368.

Sinatra, G.M. (2005): The "Warming Trend" in Conceptual Change Research: The Legacy of Paul R. Pintrich. Educational Psychologist 40 (2), 107115. http://www.tandfonline.com/doi/pdf/10.1207/ s15326985ep4002_5.

Steyrer H.P. (2009): Teaching principal plate tectonic processes by means of analogue modeling. - Studia Universitatis Babea-Bolyai, Geologia 54 (1), 13-16.

Strike, K.A. \& G.J. Posner (1992): A revisionist theory of conceptual change. In: Duschl, R.A. \& R.J. Hamilton (eds.): Philosophy of science, cognitive psychology, and educational theory and practice. New York: State University of New York Press, 147-176.
Tapponnier P., G. Pelzer \& R. Armijo (1986): On the mechanism of collision between India and Asia. In: Coward M.P. \& A.C. Ries (eds): Collision Tectonics, Geological Society London, Special Publications 19, 113-157.

Unterbruner U. \& S. Hilberg (2012): Zwischen Regenwolke und Wasserhahn. Ein interaktives Lernprogramm für Jugendliche von 10-15 Jahren. CD-ROM, Salzburg: Eigenverlag.

Unterbruner U., S. Hilberg \& I. Schiffl (2015): Understanding groundwater - students' preconceptions and conceptual change by a theory-guided multimedia learning program. Hydrology Earth System Sciences. Discussion 12, C5373-C5376. 